\title{
Electromagnetic Analysis of Graphene Nanoparticles Operating in the TeraHertz Band
}

\author{
Renato Iovine, Richard Tarparelli, Lucio Vegni \\ Department of Engineering, University of Roma Tre, Rome, Italy \\ Email: renato.iovine@uniroma3.it, richard.tarparelli@uniroma3.it, vegni@uniroma3.it
}

Received 17 May 2014; revised 18 June 2014; accepted 11 July 2014

Copyright @ 2014 by authors and Scientific Research Publishing Inc.

This work is licensed under the Creative Commons Attribution International License (CC BY). http://creativecommons.org/licenses/by/4.0/

(c) (i) Open Access

\begin{abstract}
In this work electromagnetic properties of a new type of graphene nanoparticles are investigated. The particles consist of graphene circular and square rings, embedded in a dielectric environment. The electromagnetic behavior in terms of resonant frequency position, magnitude and amplitude width for the absorption cross-section and the near electric field distribution are evaluated. Moreover, the influence of the geometrical parameters is also evaluated. The electromagnetic analysis is derived through proper full-wave numerical simulations. Numerical results show that the nanoparticles can be successfully used for the development of future graphene-based antennas operating in the TeraHertz Band.
\end{abstract}

Keywords

Graphene, Nanoparticles, Nanoantennas, Nano-Communications

\section{Introduction}

In the last few years several researchers have been involved in graphene nanoparticles-based devices development, paving the way for new engineering applications. Graphene, for its unusual electrical, thermal and mechanical properties [1], has given rise to be a potential application for high speed transistor [2], solar cells [3] and for biomedical applications [4]. Furthermore, a particularly promising emerging field is graphene-enable wireless communications. At these frequencies, metallic antennas of few micrometers are not a good solution because the resonant frequency is obtained at higher frequencies (i.e. in infrared and optical regions). Instead, electromagnetic properties of graphene material with dimensions of a few micrometers present the possibility to obtain a resonant frequency in the TeraHertz band, and high radiation efficiency [5]. 
Therefore, several researchers have investigated novel designs of nanoparticles, and all these results will prove useful in the design of future graphene-based nanoparticles for wireless nanoscale communications. For example, in [6] the authors investigate common shapes of graphene nanoparticles (i.e., rectangular and circular geometries) in TeraHertz band. They conclude that the absorbing cross section presents maximum values when the electric field polarization of the incident plane wave is tangential to the antenna's surface, and minimum values when this polarization is normal.

In this paper we present a numerical analysis of a new type of graphene nanoparticles. The particles consist of graphene circular and square rings, embedded in a dielectric environment. Compared to the classical square and circular geometries it will show the possibility to obtain a multi resonant behavior or a larger bandwidth of the absorbing cross section by varying the geometrical parameters of such nanoparticles.

The article is structured as follows: in Section 2 we present the model of the graphene nanoparticles and the full-wave numerical analysis. All the results are calculated by means of the commercial software CST studio suite [7]. Through specific numerical models, we analyze the absorbing cross-section and the near electric field distribution. Then, in Section 3 we show that the absorption of these particles, can be optimized by varying the geometrical parameters. Finally, conclusions are drawn at the end of the paper.

\section{Graphene-Based Nanoparticles}

In this Section, the electromagnetic properties of a new type of graphene nanoparticles are investigated. The proposed geometries consist of graphene circular and square rings embedded in a dielectric environment. The structure is excited by a plane-wave, having the electric field $\mathbf{E}$ parallel and the propagation vector $\mathbf{k}$ perpendicular to the plane containing the particle as depicted in Figure 1.

Specifically, the nanostructure electromagnetic properties in terms of absorption cross-sections $\left(C_{a b s}\right)$ and the near electric field distribution at the resonant frequency, are evaluated. The considered geometrical parameters are shown in Figure 1. They are: the inner radius $r_{1}$, outer radius $r_{2}$, the semi-length of inner square $l_{1}$, the semilength of outer square $l_{2}$.

Although the thickness of single layer of graphene is approximately $34 \mathrm{~nm}$ in this work we consider a multilayer graphene nanoparticles according to [8]. Therefore, in our study we have assumed the thickness equals to $300 \mathrm{~nm}$ that allows to obtain a significant intensification of the absorption cross-section spectra compared to the single layer case.

In order to study their resonant properties the following assumptions are stated:

- The particle sizes are much smaller than the operative frequency. In this way, the particles can be considered "electrically small". Therefore their resonant behavior can be studied in terms of quasi-staticapproximation. The lambda/dimension ratio equals to 12 .

- The particle is homogeneous and the surrounding dielectric environment is a homogeneous and non-absorbing medium.

Let's point out some relevant aspects concerning the present work:

- For graphene nanoparticles, Drude model of the complex permittivity function has been used [9].

- The surrounding dielectric medium is considered to be vacuum according to [10].

In this study we do not investigate the effects of different surrounding dielectric mediaon the nanoparticle electromagnetic response that are already reported in several studies [10] [11].

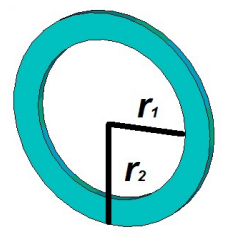

(a)
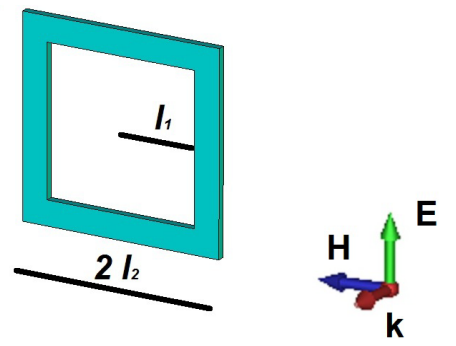

Figure 1. Geometrical sketch of the particles under study: (a)

Circular ring; (b) Square ring. 


\section{Electromagnetic Properties of Ring Graphene Nanoparticles}

We have designed the geometrical parameters of the nanoparticles in order to obtain the electromagnetic response in the TeraHertz band. More specifically, as shown in Figure 2 we have assumed the following geometrical parameters for the proposed nanoparticles: the inner radius $r_{1}=3000 \mathrm{~nm}$, outer radius $r_{2}=4000 \mathrm{~nm}$, the semi-length of inner square $l_{1}=3000 \mathrm{~nm}$, the semi-length of outer square $l_{2}=4000 \mathrm{~nm}$. The thickness of the particles equals to $300 \mathrm{~nm}$.

As shown in Figure 2, the ring particles allow to obtain an additional resonant frequency (peak II-3 THz for a circular ring and $3.2 \mathrm{THz}$ for a square ring) compared to the classical square and circular particles (peak I-0.8 $\mathrm{THz}$ ), which is in the range of the wavelength for wireless nano-communications. In addition to this, Figure 3 shows the near electric field intensification at the resonant frequency (peak II), and how the electromagnetic field is distributed on the nanoparticle surface.

In order to optimize the structure for specific applications, it is necessary to know the dependence of the electromagnetic response to the variation of geometrical parameters. Therefore in the following Section we analyze the effect of the geometrical parameters in the absorption cross-section spectra.

\section{Effect of the Geometrical Parameters}

The nanoparticles electromagnetic properties are highly dependent on the size and shape. Therefore, in this section the effect of the geometrical parameters on the nanoparticle resonant behavior is studied.

The absorption cross-section spectra presented in Figure 4 show an example of the effects of the geometrical parameters variation on graphene ring nanoparticles response. In particular, by decreasing the inner radius $r_{1}$

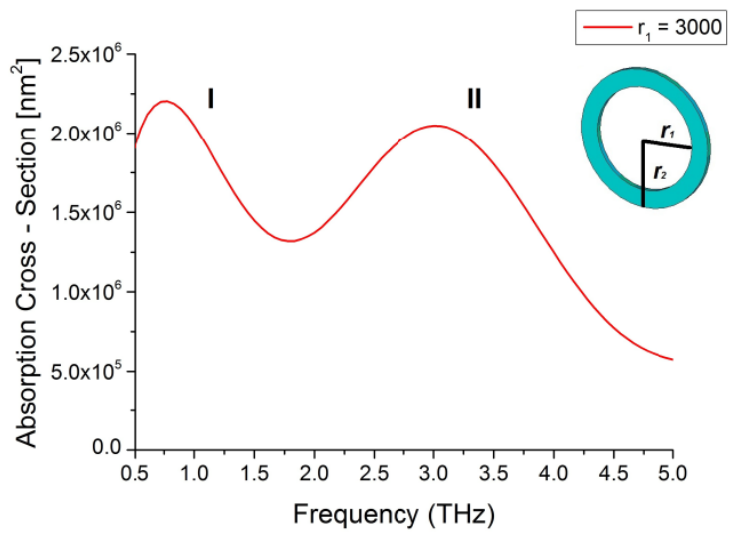

(a)

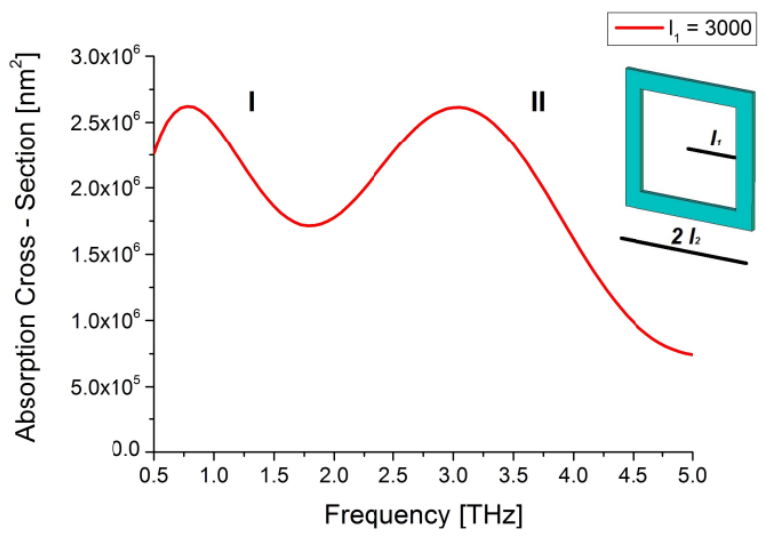

(b)

Figure 2. Absorption cross-section spectrum for (a) circular ring with $r_{1}=3000$ and $r_{2}=4000$, and (b) square ring with $l_{1}=$ 3000 and $l_{2}=4000$.

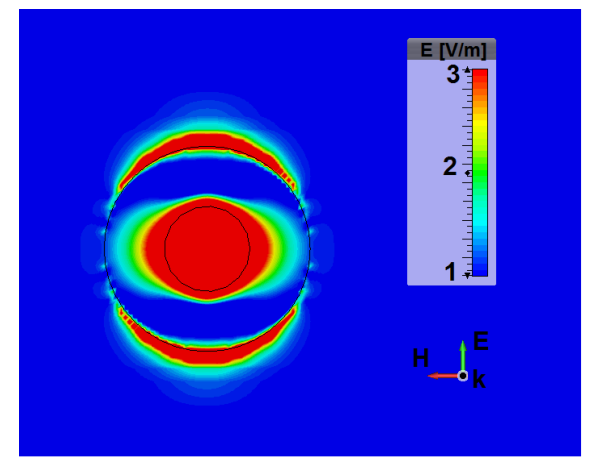

(a)

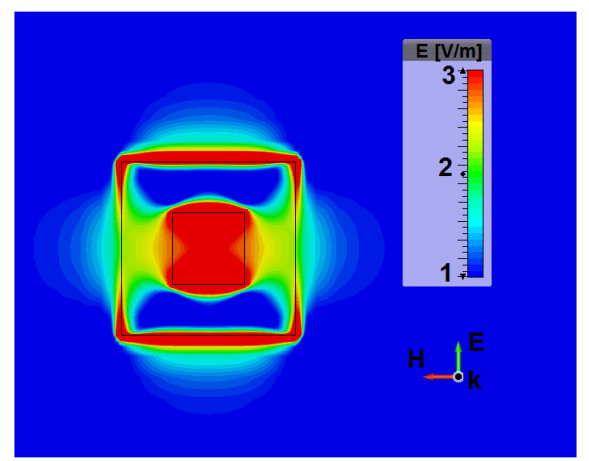

(b)

Figure 3. Near electric field distribution of (a) circular ring at $1.5 \mathrm{THz}$ with $r_{1}=1661$ and $r_{2}=$ 4000 , and (b) square ring at $1.4 \mathrm{THz}$ with $l_{1}=1661$ and $l_{2}=4000$. 
of the circular ring or the semi-length of inner square $l_{1}$ for the square ring it is possible to increase the magnitude of the spectral signal.

As reported in Figure 4(a) and Figure 4(b) for $r_{1}=l_{1}=1661 \mathrm{~nm}$ it is possible to obtain a large bandwidth signal from $1 \mathrm{THz}$ to $2.5 \mathrm{THz}$ suitable for wireless nano-communication in THz band.

In Figure 5 a comparison between the spectra of square and circular ring graphene nanoparticles is reported. As previously analyzed in [6], also for the ring particles the square shapes allow to obtain a higher magnitude compared to the circular one.

The above parametric analysis of the resonant response of graphene nanoparticles could be used to design wireless nanosystems in the TeraHertz band.

\section{Conclusion}

In this paper, a new type of graphene nanoparticles has been presented. The particles are constituted of graphene circular and square rings, embedded in a dielectric environment. The electromagnetic properties of the proposed particles have been shown in terms of absorption cross-section spectra and near electric field distribution. Extensive numerical analysis has been conducted through full-wave simulations. Furthermore, it was shown the possibility to obtain a multi resonant behavior or a large bandwidth of the absorbing cross-section spectrum by

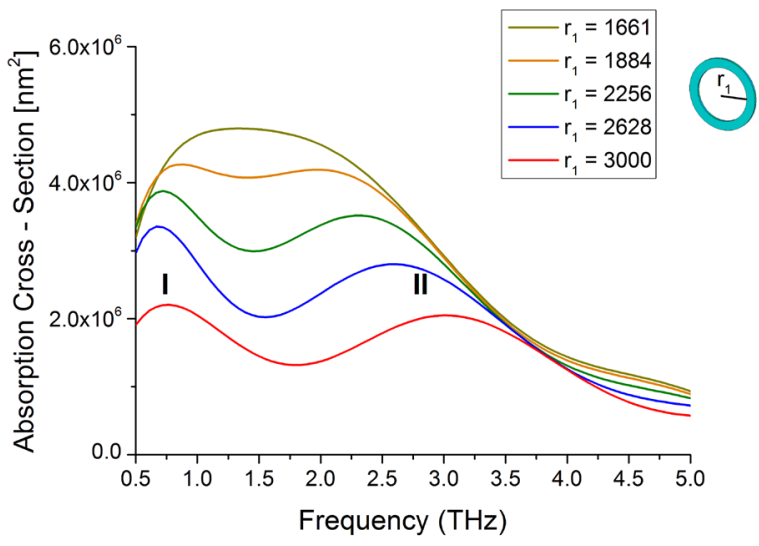

(a)

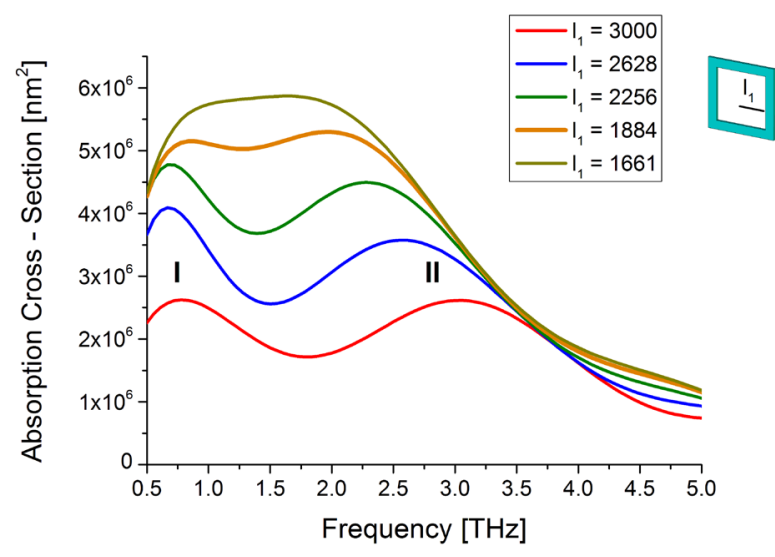

(b)

Figure 4. Absorption cross-section spectra for (a) circular ring by vaying $r_{1}$ with $r_{2}=4000$, and (b) square ring by varying $l_{1}$ with $l_{2}=4000$.

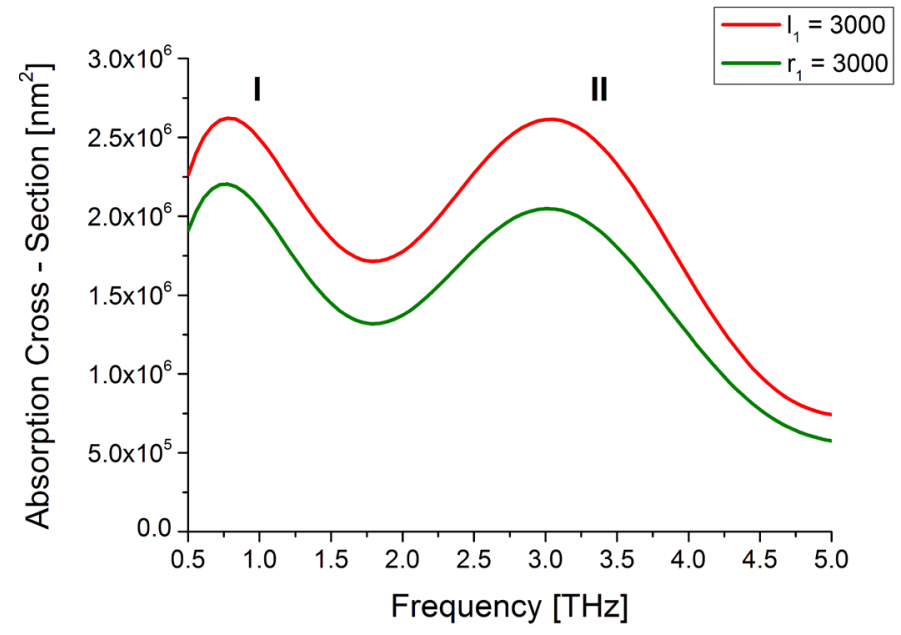

Figure 5. Absorption cross-section spectra for circular ring (green color) with $r_{1}=3000$ and $r_{2}=4000$ and square ring (red color) with $l_{1}$ $=3000$ and $l_{2}=4000$. 
varying the geometrical parameters of graphene nanoparticles. Numerical results confirm that the proposed particles could be efficiently applied for wireless communications in the TeraHertz band.

\section{References}

[1] Lee, W., Lee, J.U., Jung, B.M., Byun, J.-H., Yi, J.-W., Lee, S.-B. and Kim, B.-S. (2013) Simultaneous Enhancement of Mechanical, Electrical and Thermal Properties of Graphene Oxide Paper by Embedding Dopamine. Carbon, 65, 296-304. http://dx.doi.org/10.1016/j.carbon.2013.08.029

[2] Liao, L., Lin, Y-C., Bao, M., Cheng, R., Bai, J., Liu, Y., Qu, Y., Wang, K.L., Huang, Y. and Duan, X. (2010) HighSpeed Graphene Transistors with a Self-Aligned Nanowire Gate. Nature, 467, 305-308. http://dx.doi.org/10.1038/nature09405

[3] Bi, H., Zhao, W., Sun, S., Cui, H., Lin, T., Huang, F., Xie, X. and Jiang, M. (2013) Graphene Films Decorated with Metal Sulfide Nanoparticles for Use as Counter Electrodes of Dye-Sensitized Solar Cells. Carbon, 61, 116-123. http://dx.doi.org/10.1016/j.carbon.2013.04.075

[4] Tarparelli, R., Iovine, R., La Spada, L. and Vegni, L. (2014) Surface Plasmon Resonance of Nanoshell Particles with PMMA-Graphene Core. COMPEL, to be Published.

[5] Gomez-Diaz, J.S. and Perruisseau-Carrier, J. (2012) Microwave to THz properties of Graphene and Potential Antenna Applications. Proceedings of ISAP2012, Nagoya, 29 October-2 November 2012, 1-3.

[6] Costa, K.Q., Dmitriev, V., Nascimento, C.M. and Silvano, G.L. (2013) Graphene Nanoantennas with Different Shapes. Proceedings on Microwave \& Optoelectronics conference (IMOC), 2013 SBMO/IEEE MTT-S International, Rio de Janeiro, 4-7 August 2013, 1-5.

[7] CST Computer Simulation Technology. www.cst.com

[8] Yoon, S.-M., Choi, W.M., Baik, H., Shin, H.-J., Song, I., Kwon, M.-S., Bae, J.J., Kim, H, Lee, Y.H. and Choi, J.-Y. (2012) Synthesis of Multilayer Graphene Balls by Carbon Segregation from Nickel Nanoparticles. ACS Nano, 6, 68036811. htttp://dx.doi.org/:10.1021/nn301546z

[9] Hanson, G. (2008) Dyadic Green's Functions for an Anisotropic, Non-Local Model of Biased Graphene. IEEE Transactions on Antennas and Propagation, 56, 747-757. http://dx.doi.org/10.1109/TAP.2008.917005

[10] Llatser, I., Kremers, C., Cabellos-Aparicio, A., Jornet, J.M., Alarcón, E. and Chigrin, D.N. (2012) Graphene-Based Nano-Patch Antenna for Terahertz Radiation. Photonics and Nanostructures_Fundamental and Applications, 10, 353358. http://dx.doi.org/10.1016/j.photonics.2012.05.011

[11] Llatser, I., Kremers, C., Chigrin, D.N., Jornet, J.M., Lemme, M.C., Cabellos-Aparicio, A. and Alarcon, E. (2012) Characterization of Graphene-Based Nano-Antennasin the Terahertz Band. Proceedings on Antennas and Propagation (EUCAP), Prague, 26-30 March 2012, 194-198. 
Scientific Research Publishing (SCIRP) is one of the largest Open Access journal publishers. It is currently publishing more than 200 open access, online, peer-reviewed journals covering a wide range of academic disciplines. SCIRP serves the worldwide academic communities and contributes to the progress and application of science with its publication.

Other selected journals from SCIRP are listed as below. Submit your manuscript to us via either submit@scirp.org or Online Submission Portal.
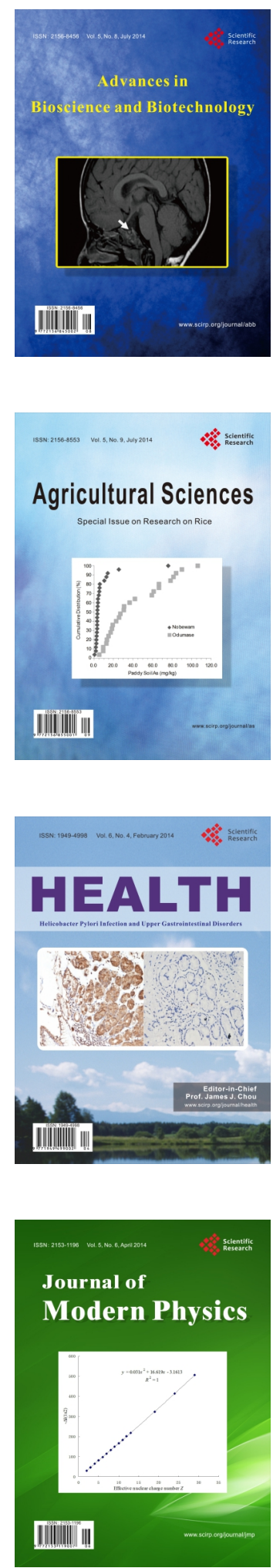
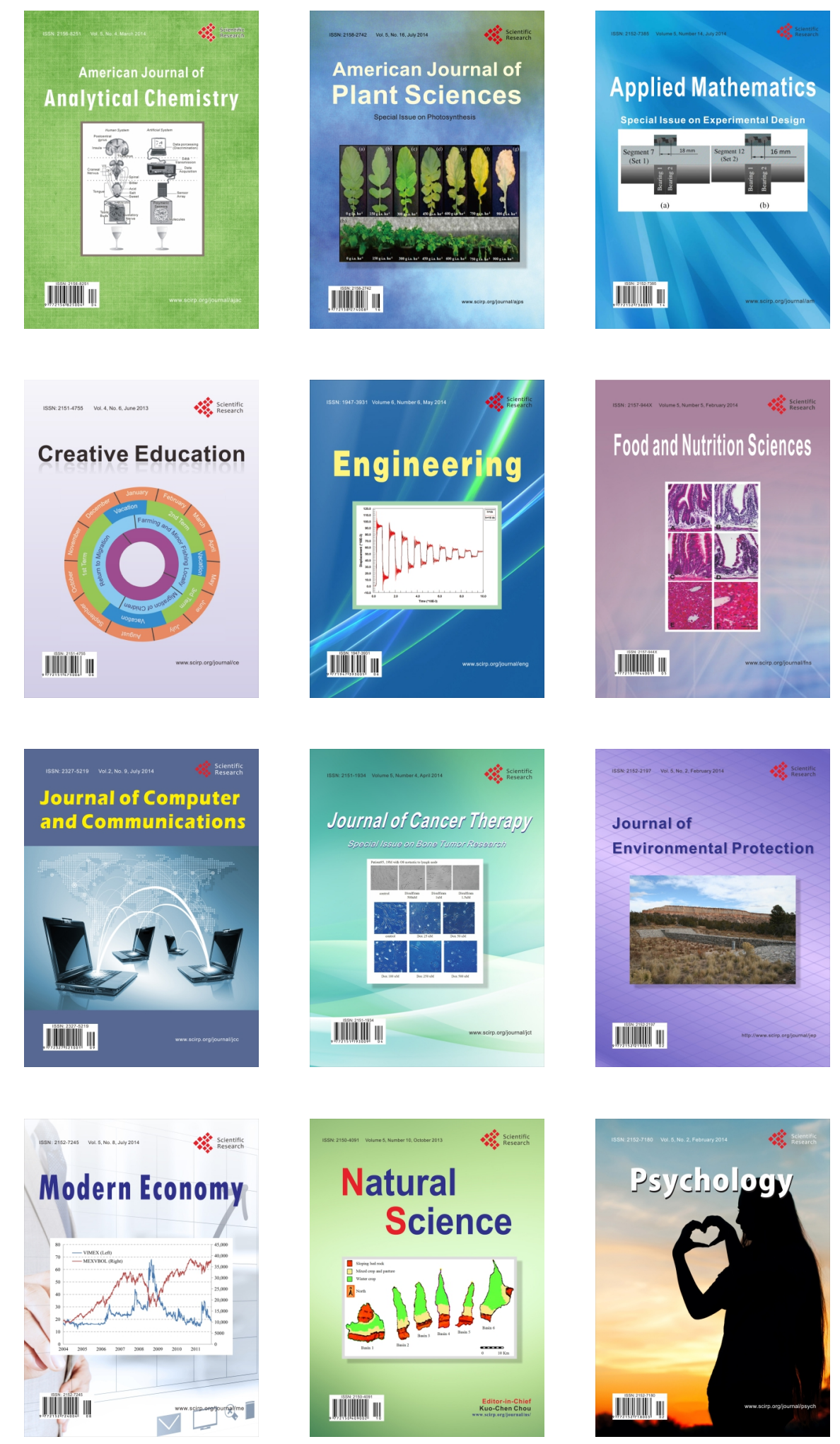